PNL-SA- -20684

DÉ93 009473

\title{
Productivity Increases in Science
}

\author{
J. K. Young \\ P. M. Molton \\ J. A. Dirks \\ J. E. Danko, Editor
}

J. J. Eberhardt, Project Manager

U.S. Department of Energy

February 1993

Prepared for

the U.S. Department of Energy

under Contract DE-AC06-76RLO 1830

Pacific Northwest Laboratory

Richland, Washington 99352

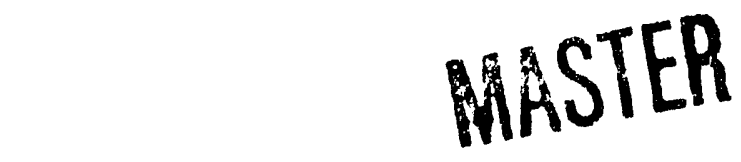




\section{Summary}

The study quantifies the impact on the cost of experimentation of synergistic advancements in instrumentation, theory, and computation over the last two decades. The study finds that the productivity of experimental investigation (experimental results $/ \$$ ) is increasing as science is transformed from a linear, isolated approach to a hierarchical, multidisciplinary approach. Developments such as massively parallel processors coupled with instrumental systems with multiple probes and diverse data analysis capabilities will further this transformation and increase the productivity of scientific studies.

The complexities and scale of today's scientific challenges are much greater than in the past, however, so that the costs of research are increasing. Even though science is much nore productive in terms of the experimental results, the challenges facing scientific investigators are increasing at an even faster pace. New approaches to infrastructure investments must capitalize on the changing dynamics of research and allow the scientific community to maximize gains in productivity so that complex problems can be attac'. .d cost-effectively. Research strategies that include user facilities and coordinated experimental, computational, and theoretical research are needed. 


\section{Acknowledgments}

The authors attribute the concepts evaluated in this document to Dr. James J. Eberhardt, Director of the U.S. Department of Energy's Office of Transportation Materials. His dedication to improving research productivity through the integration of experimental, computational, and theoretical methods began in the early 1980s; this dedication resulted in several publications and a federal program in Materials by Design in 1986. Efforts on this valuable research endeavor continue today to accelerate the development of new transportation materials.

The authors gratefully acknowledge the following contributors for their review and comment: Dr. Gary Forrest, Laser Focus World magazine; Dr. Steven Smallcombe, Varian Instruments NMR Division; Dr. Vince Dauciunas and Dr. James Crabtree, Hewlett-Packard Scientific Instruments Division; Dr. John Gurley, Digital Instruments, Inc.; Dr. J. M. Cowley, Physics Department, Arizona State University; Dr. Barry Olafson, BioDesign, Inc.; Dr. Robert Mahan, Dr. Gregory , Jxarhos, Dr. William Currie, and Dr. Mike Thompson of Pacific Northwest Laboratory; Dr. Rajat Sen of R. K. Sen \& Associates; and Dr. Enrico Clementi of IBM, Kingston, New York. 


\section{Acronyms}

$\begin{array}{ll}\text { AFM } & \text { atomic force microscope } \\ \text { EM } & \text { electron microscopy } \\ \text { FT-IR } & \text { Fourier Transform infrared spectroscopy } \\ \text { I/O } & \text { input/output } \\ \text { MBD } & \text { Materials by Design } \\ \text { MFLOPS } & \text { million floating operations per second } \\ \text { MRI } & \text { magnetic resonance imaging } \\ \text { NMR } & \text { nuclear magnetic resonance } \\ \text { NSF } & \text { National Science Foundation } \\ \text { R\&D } & \text { research and development } \\ \text { RNA } & \text { ribonucleicacid } \\ \text { STEM } & \text { scanning tunneling electron microscope } \\ \text { STM } & \text { scanning tunneling microscope } \\ \text { TEM } & \text { transmission electron microscope } \\ \text { XRD } & \text { X-ray diffraction } \\ & \end{array}$




\section{Contents}

Summary $\ldots \ldots \ldots \ldots \ldots \ldots \ldots \ldots \ldots \ldots \ldots \ldots \ldots \ldots \ldots \ldots \ldots \ldots \ldots$

Acknowledgments $\ldots \ldots \ldots \ldots \ldots \ldots \ldots \ldots \ldots \ldots \ldots \ldots \ldots \ldots \ldots$ iii

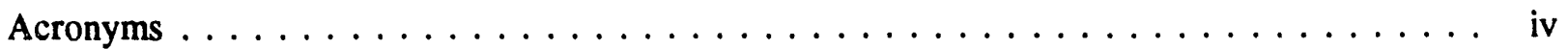

Increased Productivity of Experimentation $\ldots \ldots \ldots \ldots \ldots \ldots \ldots \ldots$

Productivity Impact of Advances in Computation $\ldots \ldots \ldots \ldots \ldots \ldots$

The Next Decade: Impacts of Massively Parallel Processing $\ldots \ldots \ldots \ldots \ldots$

Theoretical Approach to Research and Development $\ldots \ldots \ldots \ldots \ldots$

Benefits of a Coordinated Experimental and Theoretical Approach $\ldots \ldots \ldots \ldots$

Implications for Allocating Scientific Resources $\ldots \ldots \ldots \ldots \ldots \ldots \ldots$

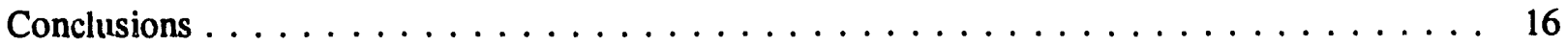

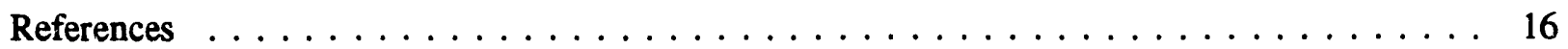




\section{Figures}

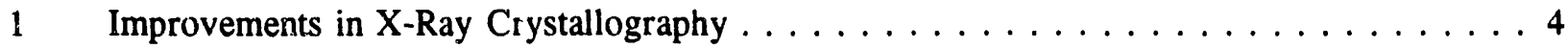

2 Improvements in Images from Electron Microscopy $\ldots \ldots \ldots \ldots \ldots \ldots$

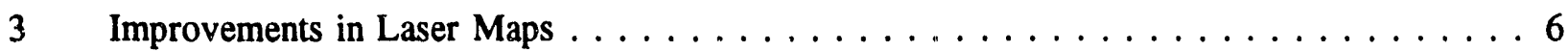

4 Improvement in Visual Quality of Data from NMR $\ldots \ldots \ldots \ldots \ldots$

5 Trends in Computer Performance $\ldots \ldots \ldots \ldots \ldots \ldots \ldots$

6 Materials-by-Design Hierarchy $\ldots \ldots \ldots \ldots \ldots \ldots \ldots \ldots \ldots \ldots \ldots$

7 Cost of Instrument Use Versus Hours of Operation $\ldots \ldots \ldots \ldots$

\section{Tables}

1 Case Studies for Documenting Improvements in Productivity of Experimentation $\ldots \ldots \ldots 2$

2 Ratio of Resources Required to Conduct an Experiment 20 Years Ago to Today . . . . . . 2

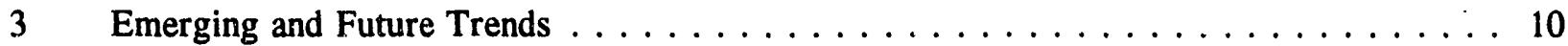

4 Case Study Experiment: $A b$ Initio Computation of a 50-Atom Molecule $\ldots \ldots \ldots \ldots$ 


\section{Productivity Increases in Science}

The results of several case studies that quantify the magnitude of productivity gains in experimental science are presented. The case studies compare the time and cost to do an experiment or simuiation today with the time and costs to conduct the same analysis twenty years ago. Finally, the study discusses the implications of the case studies and recommends research and development (R\&D) approaches that will maximize future increases in scientific productivity. A review of recent advancements in experimental science is provided.

\section{Increased Productivity of Experimentation}

Experimental science has undergone drama:ic changes because of advancements in instrumentation. Some of the most significant advancements in instrumentation over the last decade are listed below.

- A proliferation of new laser-based techniques has expanded research into time and space domains not accessible to other techniques. The most significant development in recent times is ultrafast laser spectroscopy, which makes it possible to study phenomena occurring on the femtosecond timescale (Zewail and Bernstein 1988). In addition, the increased tunability of lasers is allowing three-dimensional analysis of complex, nonhomogeneous mixtures such as flames or explosions.

- The resolution of electron microscopy (EM) has been extended (especially for biological samples) to tenths of Angstroms as a result of the development of the scanning tunneling microscope (STM) and the atomic force microscope (AFM) (Hansma et al. 1988).
- New synchrotron radiation sources (with greater intensity, near monochromaticity, and tunability) coupled with high-speed area detectors and parallel processors make possible the analysis of smaller, more dilute, more complex, and more delicate samples (Baum 1988; Maize 1988).

- Hardware advances relating to the production of a more powerful and stable magnetic field for nuclear magnetic resonance (NMR) spectroscopy are being coupled with advanced data processing capabilities. This combination allows researchers to obtain images of entire structures (Randall 1988) and to resolve the ambiguities of spectral assignments for larger proteins by increasing the dimensionality of protein NMR to four (Kay et al. 1990).

The impact of such advancements on the productivity of experimental science is illustrated in five case studies. Five types of experimental techniques were selected from a previous assessment of experimental tools (Broach et al. 1986): EM, X-ray diffraction (XRD), laser Raman spectroscopy, NMR, and Fourier Transform infrared spectroscopy (FT-IR). These instruments were selected for case studies because each represents a different type of experimental probe.

To quantify increased scientific productivity, a milestone experiment was selected from approximately twenty years ago. The time and costs to conduct the milestone experiment originally are compared with the time and costs to conduct the experiment today (in both cases, state-of-the-art equipment is assumed). The experiments selected for case studies are shown in Table 1.

Evaluating the case studies allowed the productivity increases shown in Table 2 to be 
Table 1. Case Studies for Documenting Improvements in Productivity of Experimentation

Techniques

Electron Microscopy

Nuclear Magnetic Resonance

X-Ray Diffraction

Infrared Spectrometry

Lasers
Case Study

Atomic Resolution Titanium-Niobium

Oxide (lijima 1971)

Carbon-13 Structure of Cholesterol

(Reich et al. 1969)

Crystal Structure of Lysozyme

(Blake et al. 1965)

Single Pure Compound Infrared Spectrum

Ultrafast Spectrum of Hemoglobin/Oxygen

Reaction (Greene et al. 1978)

Table 2. Ratio of Resources Required to Conduct an Experiment 20 Years Ago to Today

Productivity Impact Ratios

$\begin{array}{lrrr}\text { Technique } & \text { Experiment Time } & \text { Labor Hours } & \text { Cost }(91 \$) \\ \text { Electron Microscopy } & & & \\ \quad \text { TEM } & 180: 1 & 40: 1 & 9.9: 1 \\ \quad \text { STEM } & 7200: 1 & 1800: 1 & 2900: 1 \\ \text { X-Ray Crystallography } & & & \\ \quad \text { Conventional } & 10: 1 & 10: 1 & 21: 1 \\ \quad \text { Synchrotron } & 14: 1 & 15: 1 & 39: 1 \\ \text { Lasers } & 0.8: 1 & 1.0: 1 & 0.7: 1 \\ \text { NMR } & 9.0: 1 & 8.4: 1 & 7.3: 1 \\ \text { FT-IR } & 4000: 1 & 20: 1 & 29: 1\end{array}$

calculated for selected experimental techniques. ${ }^{\text {(a) }}$ The productivity impacts (start-tofinish experiment time, labor hours, and total cost) are shown as the ratio between the resources required to conduct the milestone experiment and the same experiment conducted today.

(a) Eberhardt, J. J., J. K. Young, P. M. Moiton, and J. A. Dirks. 1991. Technological Advances in Experimentation and Computation: Impacts on $R \& D$ Productivity. Draft Report. Pacific Northwest Laboratory, Richland, Washington.
The ratios show an improvement of one to three orders of magnitude in almost all productivity areas. The single exception is the laser probe. The laser is still a laboratory tool assembled by the researcher (with much of the labor directed toward set-up time). The price of laser systems, even in constant dollars, has approximately doubled because of the much greater stability required to achieve narrower line widths, shorter pulses, and/or new wavelength ranges. These advancements in laser technology have allowed many more intermediate data points to be collected (the duration of 
the laser pulse today is about 60 times shorter than in the milestone experiment). Hence, although the actual experiment time and cost have increased somewhat, the data are of much higher quality.

While direct comparisons of productivity using similar instruments typically show substantial improvement over time, new analytical techniques for which there are no historical equivalents show even greater productivity improvements for two of the case studies. For example, using a scanning tunneling electron microscope (STEM) rather than a transmission electron microscope (TEM) to perform the milestone electron microscopy experiment increases all the productivity impact ratios by more than an order of magnitude. Similarly, the synchrotron light source also shows a notable improvement over the conventional technology in the X-ray uistallography experiment.

Examples of the increased capabilities for visualizing results for the case study instruments are shown in Figures 1 to 4 . In these figures, spectra of what could be obtained as little as ten years ago are compared with more recent visual interpretation of spectra obtained using state-ofthe-art instruments and new advanced software for collecting and interpreting the data. These figures illustrate the improved quality of $d$ ita that can be collected today within a much shorter time period.

\section{Productivity Impacts of Advances in Computation}

Integration with the computer, with its vast capacity for storing data and executing calculations, has played a major role in increasing the productivity of sophisticated scientific instruments. Manufacturer's hardware improvements to instruments are attributed to synergistic developments in computing; for example, increased instrument stability, focus, and beam control result from the computer control of fabrication that allows the design and machining of much smaller and more intricate parts for instruments. (Parts can now be machined to a precision of 0.25 micrometers; 20 years ago, 25 micrometers was the limit.) Thus, the instruments and computers of one generation allow manufacturers to build the improved instruments and computers of the next generation. ${ }^{(a)}$ In addition, dedicated, advanced data processing/integrated circuitry allows collection and transformation of much more reliable, complex, and detailed data.

The integration of dedicated, online data processing capabilities with scientific instruments would not have been possible without significant reductions in the cost of computing. Figure 5 illustrates the decrease in the computing cost/performance ratio of leading-edge computers since the 1950s. The purchase cost represents the cost when the machines were introduced (Gille Associates 1961; Lazou 1986; Dongarra 1985). The machines chosen are examples of the best technology available (Williams 1985). A range of computing costs has been calculated for each machine. For early machines, the top of the range represents the fact that addition was much faster than multiplication or division (bottom of the range). The peak speeds, with later machines using vector or parallel processors (top of the range), are much faster than the speed when the machine is being operated in scalar mode (bottom of the range).

Much of the reduction in computational costs in the past 35 years is a direct consequence of advancements in solid-state electronic devices. Since 1958 when the first solid-state electronic device was implemented to replace

(a) Eberhardt, J. J., J. K. Young, P. M. Molton, and J. A. Dirks. 1991. Technological Advances in Experimentation and Compuzation: Impacks on R\&D Productivity. Draft Report. Pacific Northwest Laboratory, Richland, Washington. 


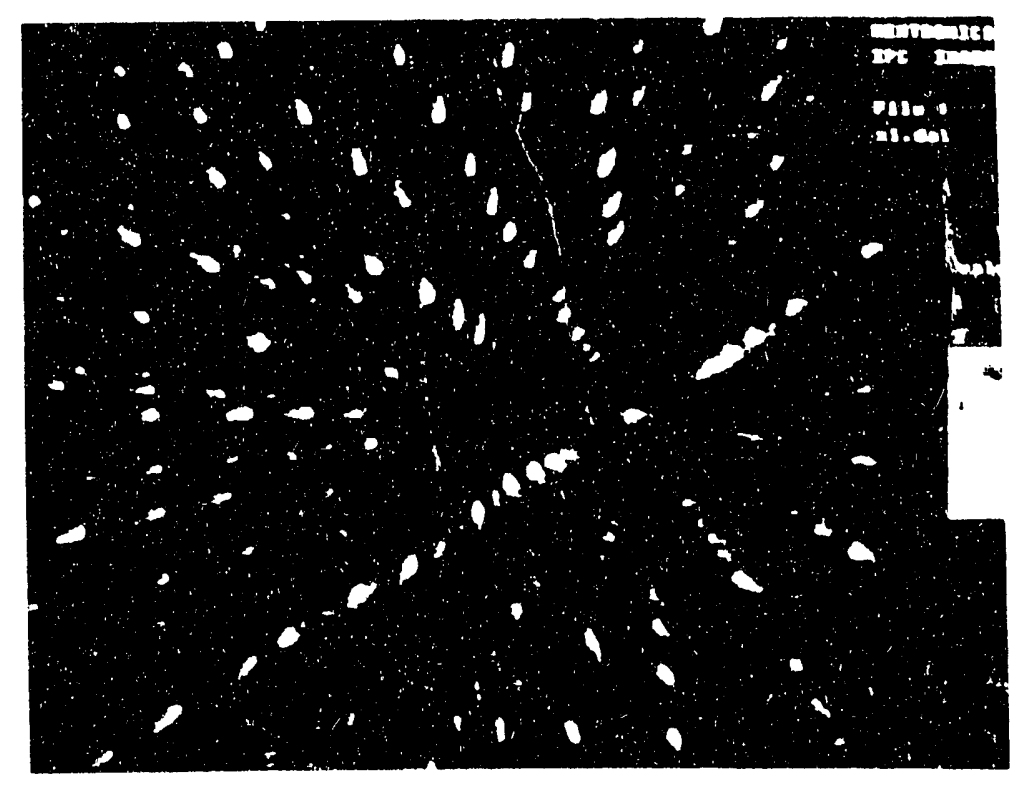

(a) Photograph of back reflection pattern; characteristic of data displayed by a 1970 instrument. ( ${ }^{\circ}$ Siemens Analytical X-Ray Instruments, Inc. Reprinted with permission.)

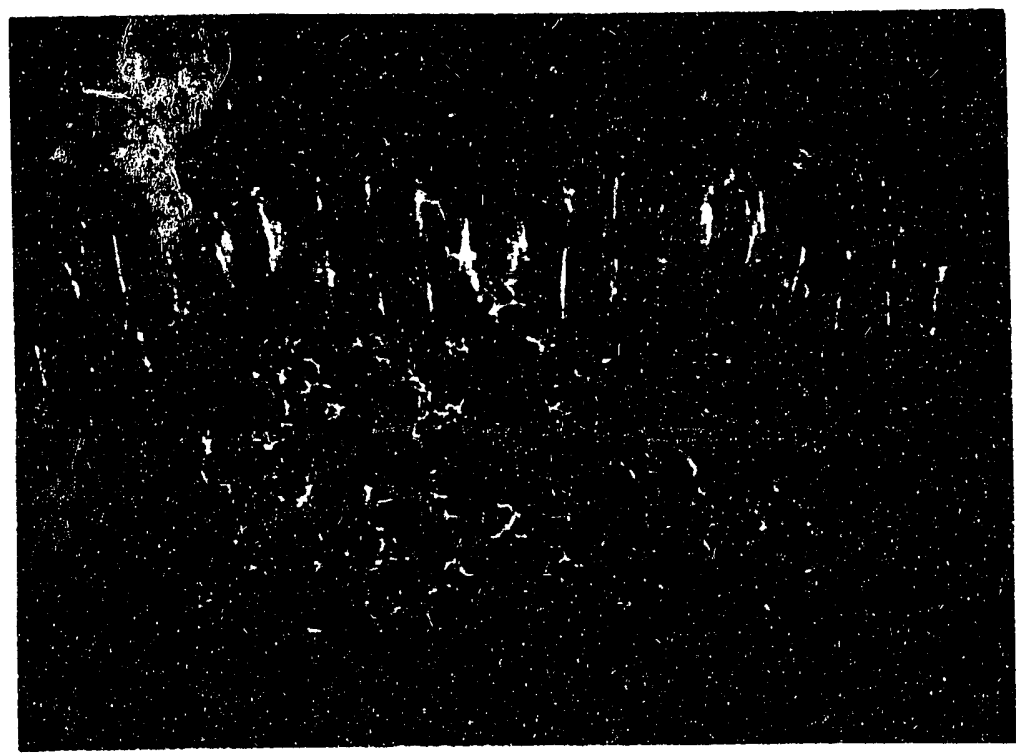

(b) X-ray diffraction image of a crystallized protein-DNA complex consisting of a DNA fragment (blue) and two protein fragments; image obtained in 1989. (A. Mondragen and S. C. Harrison, Howard Hughes Medical Institute and Department of Biochemistry, Harvard University, Cambridge Massachusetts. Reprinted with permission.)

Figure 1. Improvements in X-Ray Crystallography 


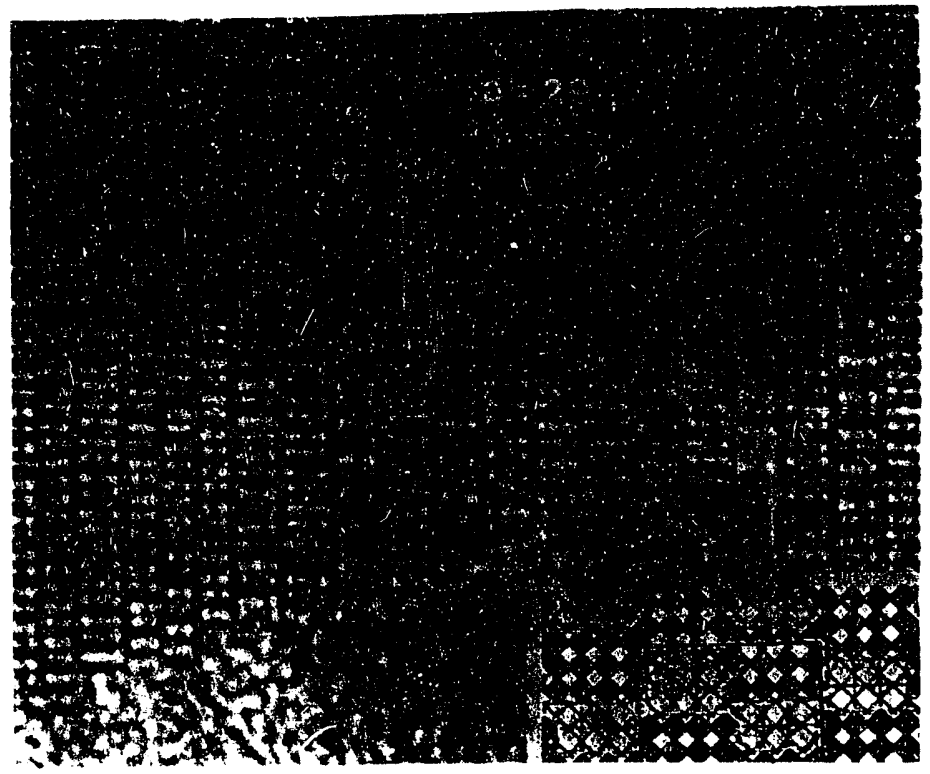

(a) Electron microscope image of a thin crystal $\mathrm{Ti}_{2} \mathrm{Nb}_{10} \mathrm{O}_{29}$. (Sumio lijima, 1971, Journal of Applied Physics 42:5891. Reprinted with permission.)

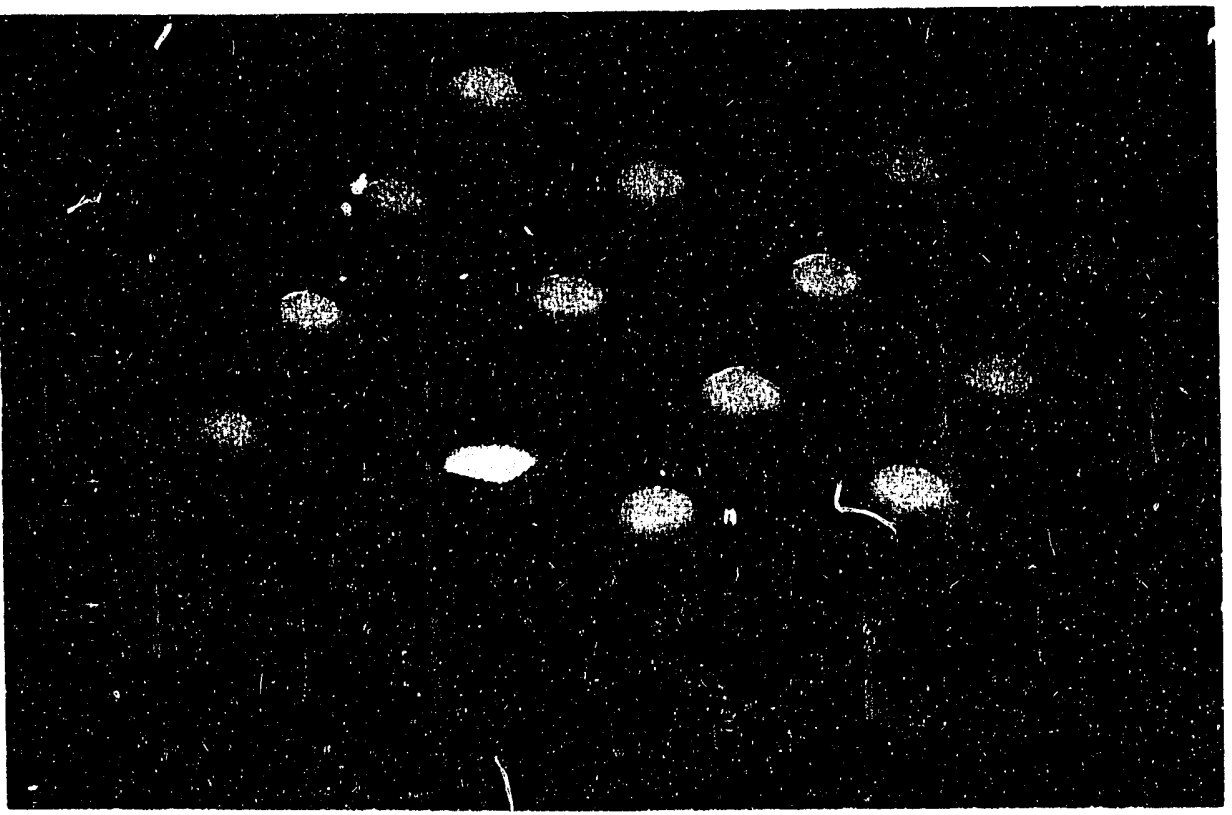

(b) Atomic imagine of iodine atoms in a $3 \times 3$ array absorbed on a platinum crystal. (Data from Dr. Bruce C. Schardt, Department of Chemistry, Purdue University, taken on a Digital Instruments Nanoscope II STM. Reprinted with permission.)

Figure 2. Improvements in Images from Electron Microscopy 


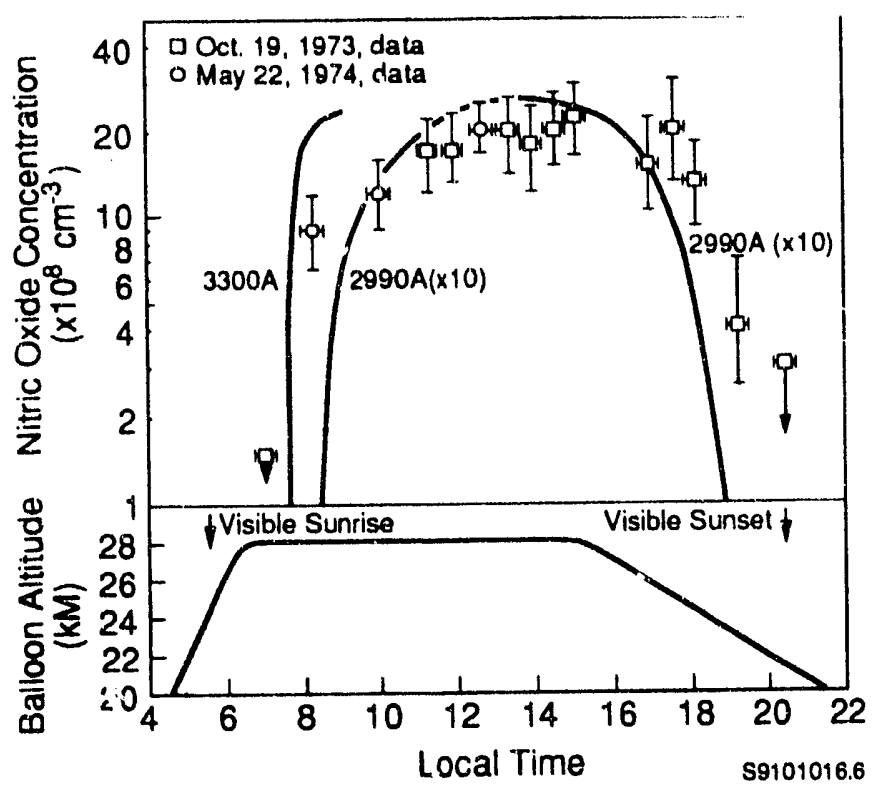

(a) Quantitative comparison of atmospheric nitric oxide concentration data as a function of time; obtained in 1974 by C. K. Patel of Bell Laboratories, New Jersey. (Reprinted with permission from Optical and Quantum Electronics, Chapman \& Hall, London.)

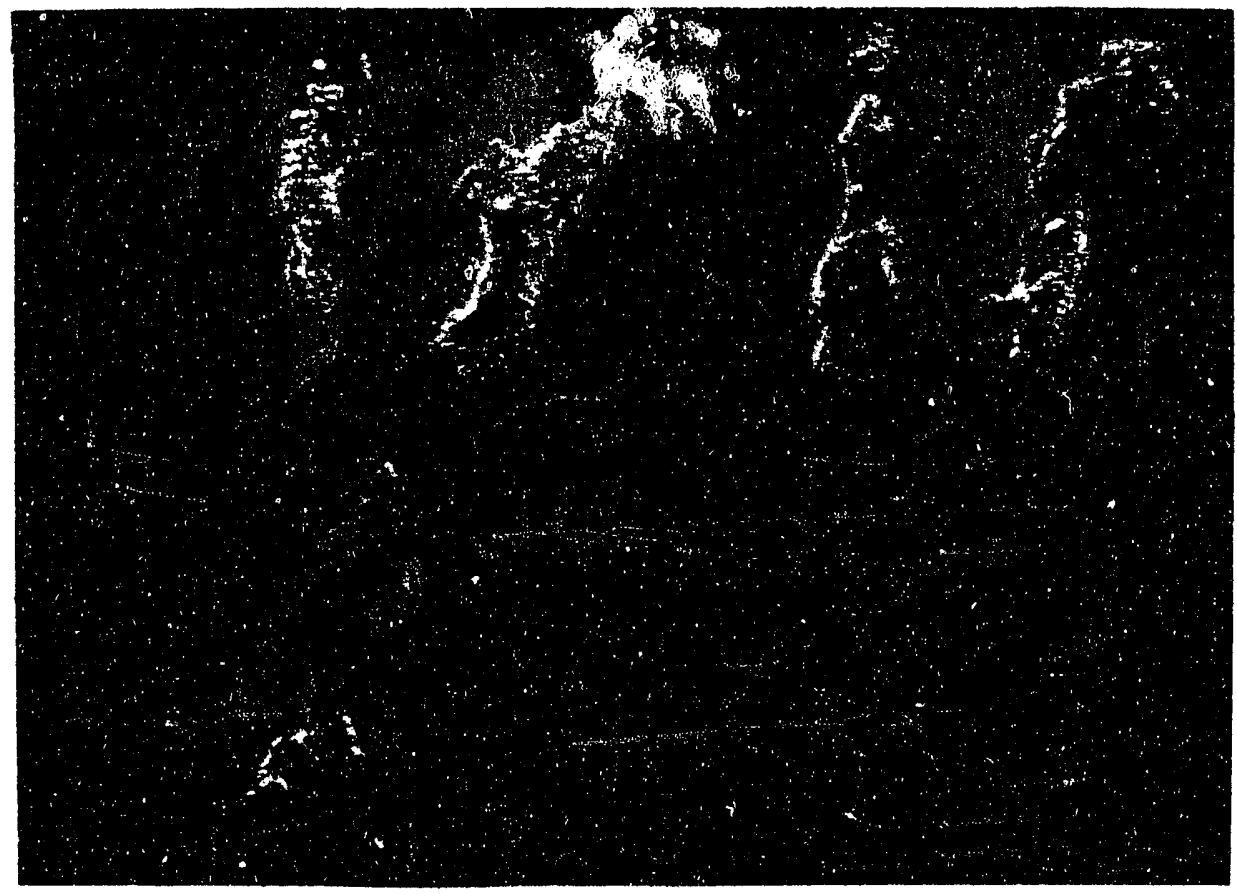

(b) Instantaneous map of fuel concentration in a turbulent diffusion flame; obtained by Dr. Marshall Long at Sandia National Laboratory in 1983.

Figure 3. Improvements in Laser Maps 


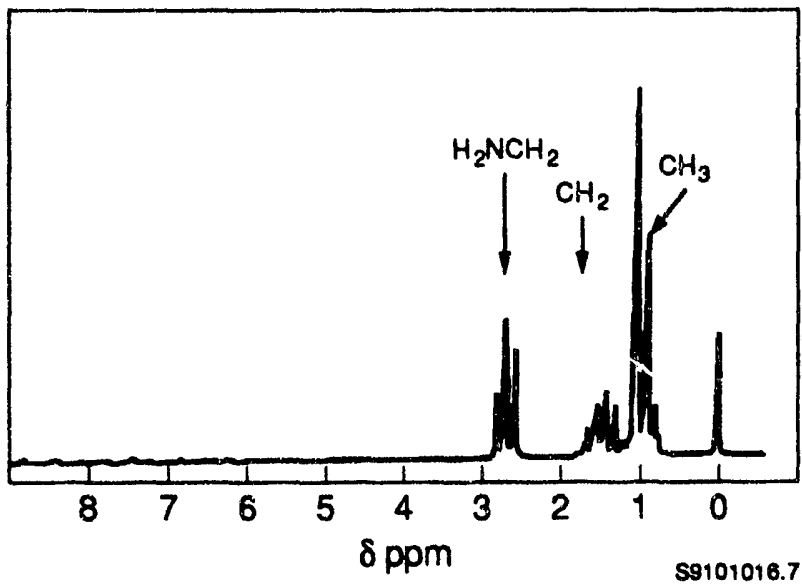

(a) Representation of NMR spectrum on 1-aminopropane (n-propylamine) in $\mathrm{CDCl}_{3}$, characteristic of 1970 technology. (D.A.R. Williams 1986. Nuclear Magnetic Resonance Spectroscopy, ACOL Series. Reprinted with permission of ${ }^{\bullet}$ John Wiley \& Sons, Inc.)

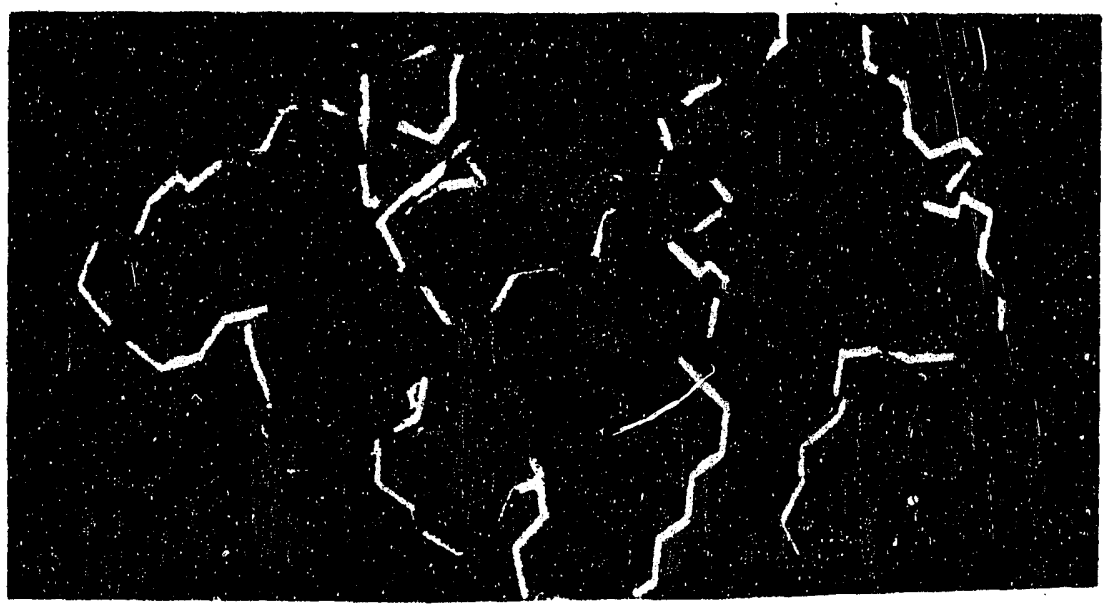

(b) The minin-chain of protein crambin (yellow) using NMRgraf structure (blue) obtained through $\mathrm{x}$-ray crystallography, 1989. (Photo by Dr. Barry D. Olafson, BioDesign, Inc., Pasadena, California. Reprinted with permission.)

Figure 4. Improvement in Visual Quality of Data from NMR 


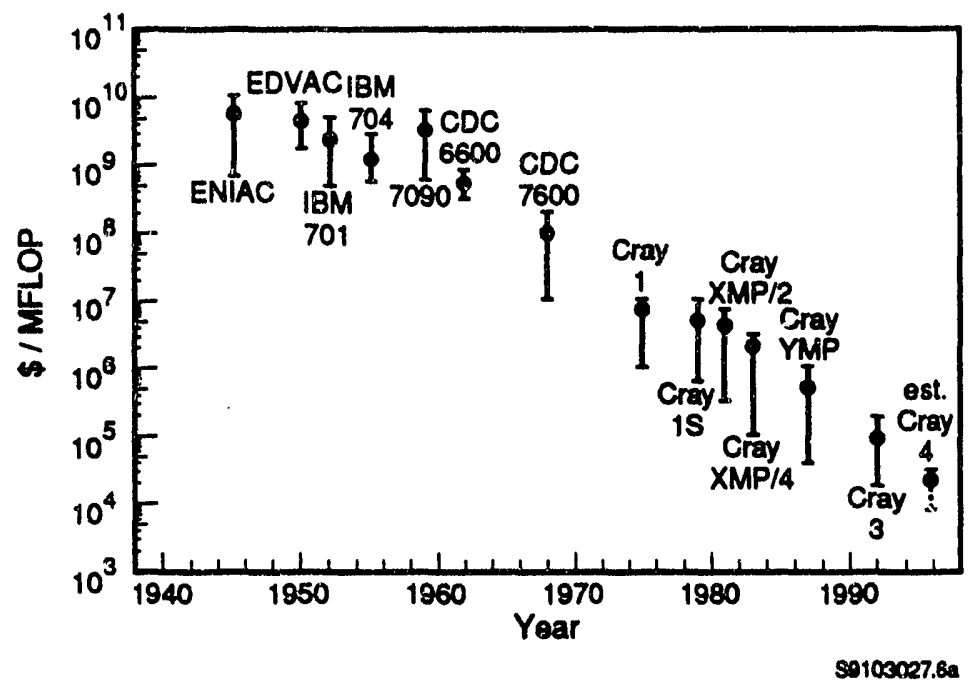

Figure 5. Trends in Computer Performance

vacuum tubes, circuit density has doubled every year or two; at the same time, chip size has decreased. Increased chip density and new computer-aided design approaches made possible the development of increasingly complex circuits to execute logic operations (Rush 1985). The increased density and sophistication of solid-state electronic devices also resulted in increased fast memory capacity and input/output bandwidth for computer processors. Because the distance an electronic message must travel between logic elements is much shorter in chips with greater density, the clock speeds for computers today are three to six orders of magnitude faster than early computers.

In the 1970s, the foundation of supercomputing machines was formed with the introduction of two new architectural features: vector processing and parallel processing. Vector and parallel processing are continuing to reduce the cost of computing.

The first highly successful vector processor was the Cray 1. The Cray 1 had a peak speed of 140 million floating operations per second (MFLOPS), almost ten times faster than earlier scalar machines. The Cray 1 enabled truly three-dimensional simulation modeling of physical problems in a reasonable amount of computer time (Berlin 1987).

The first highly successful multiprocessing supercomputers, the Cray X-MP series introduced in the early and mid-1980s, supported 2and 4-vector processors; the later Y-MP system introduced in 1988 supported 8-vector processors (National Research Council 1988).

According to staff at Cray Computer Inc., the Cray 3 system now being developed will include 8- to 16-vector processors and will use gallium arsenide (rather than silicon) semiconductor chips. Gallium arsenide semiconductor chips are ten times denser and have twice the switching speed of silicon-based semiconductors. The Cray 4 planned for the 1994-1996 time period might support up to 64-vector processors. Several other computing firms are also developing massively parallel machines for future delivery. The performance of these multiprocessor systems depends primarily on the degree to which the program can be divided into a high percentage of vectorizable and 
parallel tasks, the complexity of the calculations to be performed, and the number of processors available to execute the program (Buzbee and Sharp 1985).

\section{The Next Iacade: Impacts of Massively tarallel Processing}

Massively parallel processing will form the basis of the next decade of advances in scientific instruments. One example of a massively parallel computer currently available is The Connection Machine with 64,000 processors (National Research Council 1988). Each of these processors has relatively low speed and performance, but when taken together, they can compete with or even outperform the fastest scalar computer. Such machines are uniquely suited to several emerging computing capabilities that can be divided into a large number of work units requiring concurrent execution of simple operations; for example, vision and speech recognition; complex computer simu!ations of the interactions of atoms and molecules; and logical processing of concepts, ideas, and images (Buzbee and Sharp 1985). However, the current lack of software and operating systems represents a substantial barrier to widescale implementation of massively parallel machines.

As massively parallel processors are integrated with increasingly sophisticated experimental probes, breakthroughs in experimental productivity will continue to occur. Although few new instrumental techniques will likely be developed over the next decade, hardware and techniques for existing instruments will probably be refined and diversified. Miniaturization will follow, and instrument manufacturers will develop instruments (for many types of probes) that will allow process measurement and control both online and in the field. Dramatic developments in experimental science, such as those shown in Table 3, can be anticipated over the timeframes indicated.
In particular, the integration of massively parallel processing with instrumentation will significantly advance the scientist's ability to visualize molecular-level data in real time. Visualization is uniquely suited to massively parallel machines because each processor can be set up to carry information about a selected element in a much larger structure. This information is used to model the structure being examined on-screen. The image then can be manipulated and re-examined from a variety of viewpoints. Scientific computations involving matrix multiplication, convolution, series expansion integration, linear equations, and Fourier Transformations are other especially promising applications for massively parallel machines (Broach et al. 1986).

Over the next decade, the improved visualization and data processing capabilities of instrumentation are expected to result in other revolutionary capabilities for scientific investigation:

- Advancements in image processing (to remove background artifacts) and development of high-intensity electron beams will allow development of transmission electron microscopes that operate at ambient temperatures.

- AFM and STM probes combined with sensors of different types will allow scientists to watch chemical reactions as they occur. Coupling EM with holography will allow such phenomena as magnetic fields and electron densities to be imaged. STM probes will be used as tools to modify surfaces by moving atoms around. Realtime imaging will be used to guide the researcher in obtaining desired physical or chemical properties.

- Ultrafast laser spectrometers with expanded tunability and scanning ability will allow time-resolved, multicomponent analysis of chemical systems. 


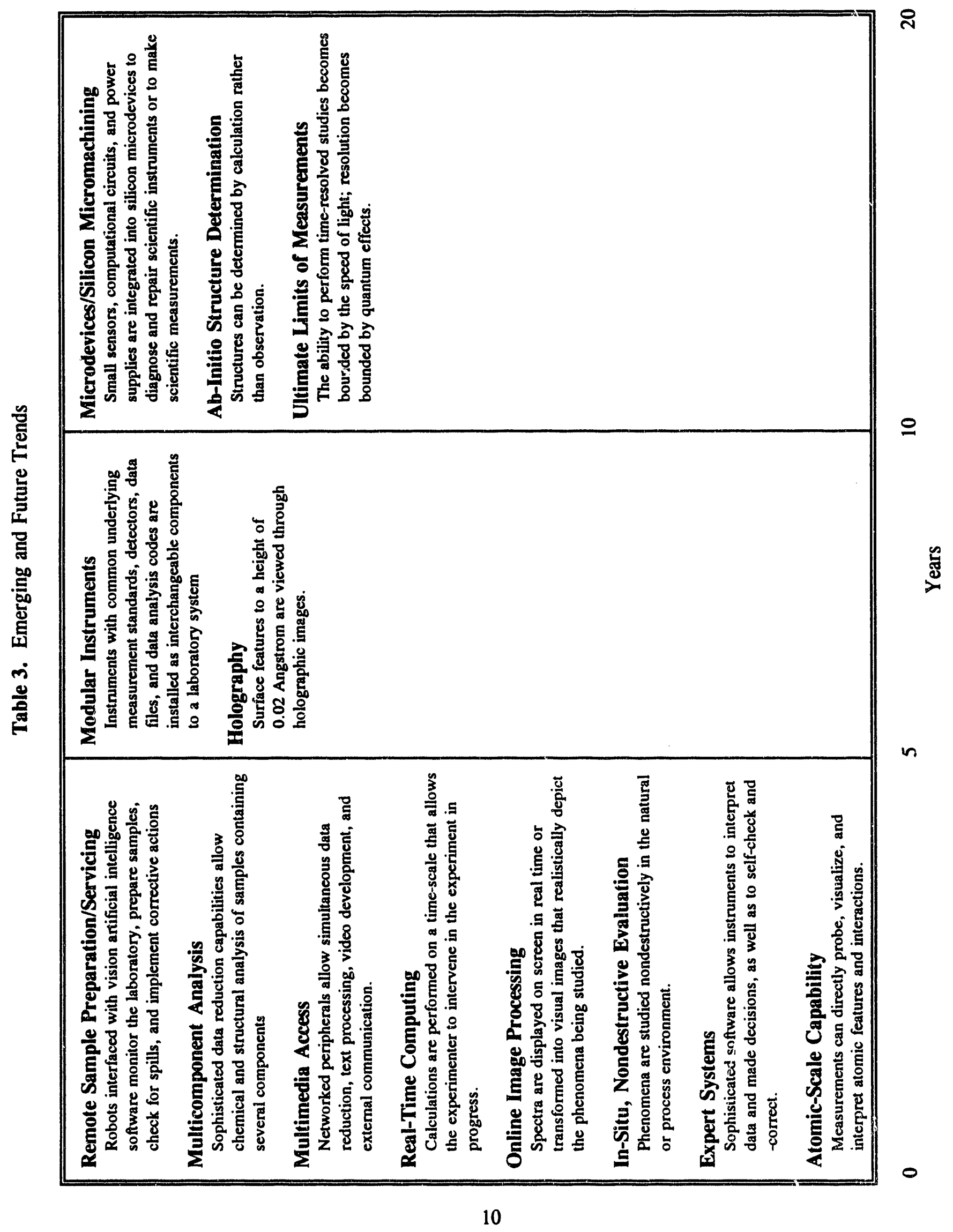


- Three-dimensional MRI imaging will no longer be used strictly for medical diagnosis, but for the study of real-time interactions within living systems. For example, Branscomb (1986) predicts that NMR imaging will allow the biochemical activity of the brain to be correlated with cognitive activity and behavior.

Another dramatic trend in computing that will transform science over the next decade is the U.S. High Performance Computing Initiative. The objective of this initiative is to provide supercomputer access to researchers from any point in the nation. This program builds upon the National Science Foundation's (NSF) supercomputing centers and provides a focal point for developments in parallel processing, high-speed networking, distribution software tools, and effective solutions to supercomputing mass storage needs. Through NSF-supported improvements to NSFnet-the network that will connect major research universities, government laboratories, and industrial laboratories-a high-speed, national, electronic superhighway system for the highspeed transfer of information (40 gigaby second) will be created. Using NSFnet wri! expand computing from an activity conducted on a single multiprocessor machine to a largescale, dynamic system of processors that spans a continent.

These trends result in many exciting implications for science. In 10 or 20 years, it is conceptually possible that scientists will be able to

- read DNA strands directly

- monitor transition state energy curves in real time and in three dimensions

- observe molecular-level reactions in living cells

- obtain single atom spectra
- couple different experiments and techniques remotely and simultaneously via computer networking.

\section{Theoretical Approach to Research and Development}

Massively parallel architectures promise to provide the low-cost computational capabilities that will allow $a b$ initio atomic simulations on a scale that approximates realistic systems. Efforts are needed, however, to integrate emerging theoretical/computational methods with experimental techniques. Such efforts should include developing a common language to facilitate the collaboration required between experimentalists and theorists to develop experimentally validated supercomputer models for simulating the nature of materials.

The speed and capabilities of $a b$ initio computer models have already advanced significantly. In 1964, IBM researchers began work on the code entitled IBMMOL to perform ab initio computations on large chemical systems. IBMMOL version I (1965) could handle up to 50 atoms; 100 contracted gaussian functions; 800 primitive s,p,d, and forbitals; and any type of geometry. Lack of fast computers precluded running the code with 50 atoms until 1972. According to Dr. Enrico Clementi, a developer of the code, running a 50-atom simulation on the IBM $360 / 75$ system required in excess of 10 hours of computer time. The capital cost of the computer was about $\$ 860,000$ (1972\$), with a speed of about 3 MFLOPS.

A simulation identical to the one Clementi ran in 1972 would likely be run on an RS6000 computer today in about 7 minutes. Ignoring the programming time and the time required to input the data and output the results, the time required for the simulation has been reduced by a factor of 80:1 (Table 4). Assuming similar annual operating hours for each computer and 
Table 4. Case Study Experiment: $A b$ Initio Computation of a 50-Atom Molecule

$$
1972 \text { IBM 360/75 } 1990 \text { RS6000 }
$$

Experiment Time:

Computer Operation

(CPU time)

10 Hours

-7.2 Minutes

Simulation Cost: ${ }^{(a)}$

$\$ 4,900$

$\$ 1.46$

Data Analysis

Productivity Impact Ratios ${ }^{(\text {b) }}$

Cost Reduction

NA

83.3:1

Experiment Time

NA

3378:1

Reduction

(a) All costs converted to 1991\$; includes equipment cost only-no labor cost assumed.

(b) Compared with 1972 simulation.

NA $=$ Not applicable.

converting all costs to $1991 \$$, the cost reduction ratio is greater than three orders of magnitude.

While theoretical modeling capabilities have increased along with computational power, there are some probiems with the current research approach. While higher computational power increases accuracy, it does not yet increase scope by simulating larger systems. For example, IBM's new code, renamed KGNMOL-IBMMOL, can perform much more sophisticated quantum-chemistry calculations. KGNMOL was recently applied to a mediumlarge molecule of a 60-atom carbon cluster. The objective was to arrive at a quantitative prediction of cluster stability, electron affinity, ionization potentials, singlet-triplet excitations, and the stability of cluster-alkali metal complexes and their geometry. The calculations were performed using the parallel version of the KGNMOL code. For the double-zeta basis set, the computation of the integrals (one- and twoelectron) required 5 hours of elapsed time; the integrals were stored on 17 triple-density magnetic disks (IBM 3380) for a total of approximately 27 gigabytes. Because the integrals were accessed in parallel, each iteration required about 90 minutes of elapsed time.

If theory is to play a role in increasing the productivity of science, a balance must be struck between the need for accuracy and the development of "good enough" simulations on systems large enough for predictive purposes. It is these predictions that will allow experimental researchers to devise efficient experimental approaches for large-scale simulations. The focus must be on alternative methods and new techniques that obtain a balance between accuracy and predictive abilities; these techniques must be capable of efficiently using advanced computers to do real-life simulations. In addition, computational chemistry must bring additional focus on developing databases, expert systems, interactive graphics with animation, and artificial intelligence. Parallelism, both coarse and massive, will be essential in this endeavor. 


\section{Benefits of a Coordinated Experimental and Theoretical Approach}

In the future, several computational-intensive capabilities will become tractable for coupling with experimental probing of time-resolved phenomena at the atomic level (e.g., transition states, grain boundary adhesion, fracture mechanisms, etc.). These investigations will provide the knowledge base for developing predictive theoretical tools that enable scientists to simulate atomic level interactions on the computer. Once a theoretical technique replicates a sufficient number of prior experimental tests, the validity of the model is established. The model can then be extended to making predictions, for example, about the structure and properties of new materials, some of which may not have been synthesized yet, at conditions other than those used in the experiment. The experimental tools are used again to verify that the material exhibits predicted properties.

As scientists use theoretical information to guide the experimental process, fundamental information critical to the development of new products and processes will be gained much more rapidly via computer simulation. Based on experimental observations, models of materials interactions at the atomic level will permit material structures and properties to be simulated on supercomputers. Ultimately, such models could be used to predict the properties of new materials. As a result, many more needed innovations in products and processes for U.S. industry would occur in a much shorter timeframe. This is the basis of the U.S. Department of Energy's "Materials by Design" (MBD) program (Eberhardt et al. 1986).

The basis for the MBD approach is a hierarchy of models (see Figure 6) to help a researcher design materials more effectively. The models span the spectrum from electronic and atomic/molecular models of a material to large systems integration models that would optimize materials performance in real systems. To build such models requires expert knowledge and cooperation from many disciplines. In addition, a multitude of experimental investigations will be needed to develop the understanding of materials interactions at the atomic/ molecular level so that such interactions can be simulated on the computer. The implications of such a program are incredible: an MBD capability would transform science from a linear, isolated activity to a much more productive, hierarchical, integrated activity.

A key rationale for DOE's support of MBD was that it would expand the technology base available for private sector development of materials important to increased energy efficiency (e.g., lightweight materials, hightemperature materials, and catalysts). To understand how the development of MBD tools might affect the catalyst $R \& D$ process, Pacific Northwest Laboratory interviewed R\&D managers from U.S. industry. The following impacts were identified as most important:

- Catalysts can be optimized because performance can be simulated under a wide variety of operating conditions.

- Undesirable candidates are eliminated before expensive experiments and tests have been conducted.

- Experimental and bench-scale studies to define the most relevant parameters will be of higher quality.

Industrial R\&D managers speculated that a theoretical approach could reduce by as much as $50 \%$ the time required to conduct exploratory research. This $50 \%$ time reduction could dramatically increase the competitiveness of U.S. industry in world markets (Fassbender et al. 1989). As the microscopic information from the lowest levels of the MBD hierarchy is integrated into the systems performance models at the highest level of the hierarchy, process 


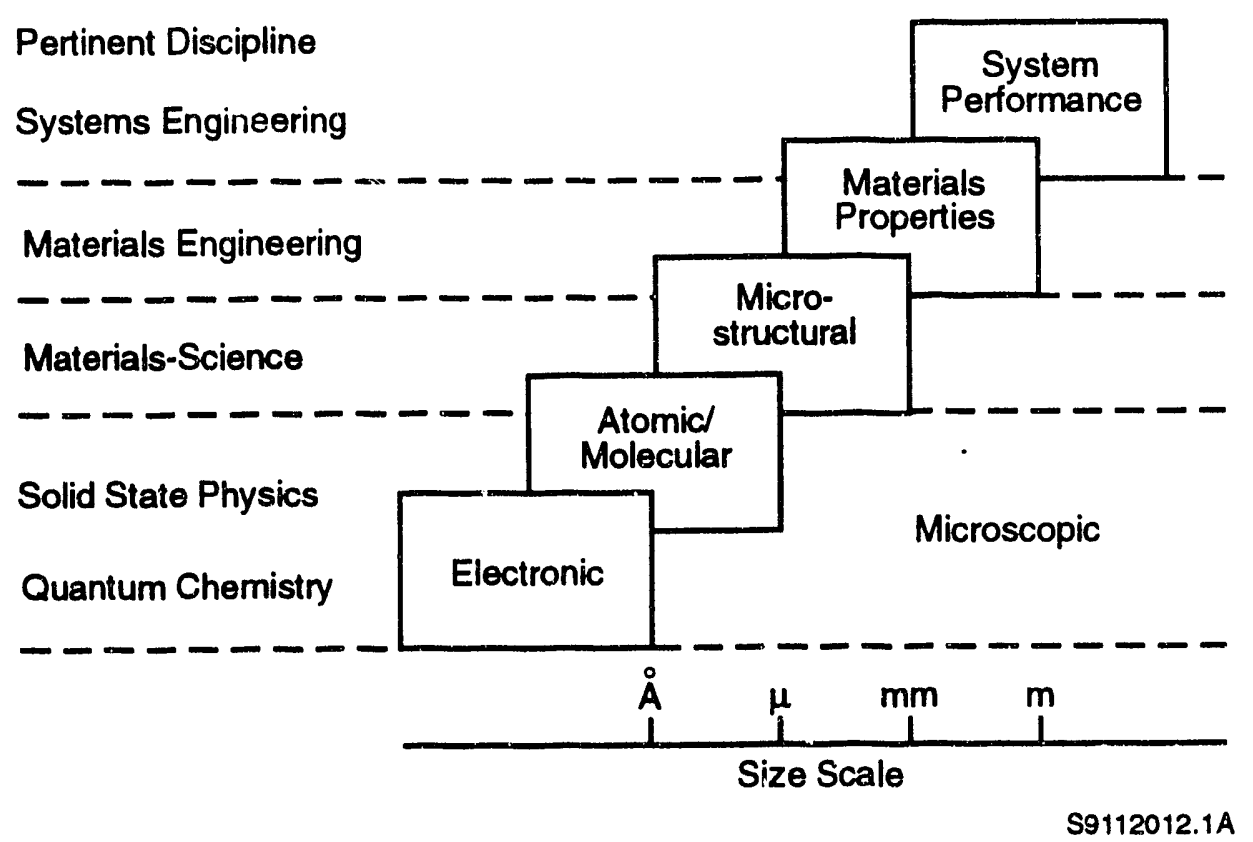

Figure 6. Materials-by-Design Hierarchy (Eberhardt et al. 1986. ${ }^{\circ}$ Materials Research Society Symposium Proceedings, Vol. 63, Pittsburgh, Pennsylvania. Reprinted with permission.)

designs for the production and use of a new material could be evaluated concurrently with exploratory research, resulting in further time and cost savings.

\section{Implications for Allocating Scientific Resources}

Given the magnitude of economic benefits from combined experimental and theoretical studies, the correct programming of R\&D funding is critical. Unfortunately, the cost of instruments and supercomputers increases with their capabilities. The increase is not due to capital costs. On a current dollar basis, the sophisticated instruments and supercomputers of today cost about as much as the sophisticated instruments of 20 years ago. However, the lifetimes are much shorter.

Figure 7 illustrates the impact of advancements in analytical instrumentation on the costs of research for two instruments, TEM and NMR. As the figure shows, the cost per hour of inst-ument use has increased significantly (as exhibited in the differences between the 1969 and 1989 lines for each instrument). These cost increases have occurred largely because the rapid changes and improvements in the technologies have reduced their useful life (i.e., the time to obsolescence is shorter). Typically, these hourly cost increases have been more than offset for the researcher by the decreased time required to conduct a given experiment, as illustrated by the difference in the annual hours of operation assumed for the 1969 and 1989 NMR spectrometers. Effective strategies for using instruments could further reduce costs.

As Figure 7 shows, the instrument should be used to the greatest extent possible to minimize the hourly cost. To make the cost per hour of use for sophisticated instruments equivalent to 20 years ago, the instruments must be used about twice as much (4000 hours per year 


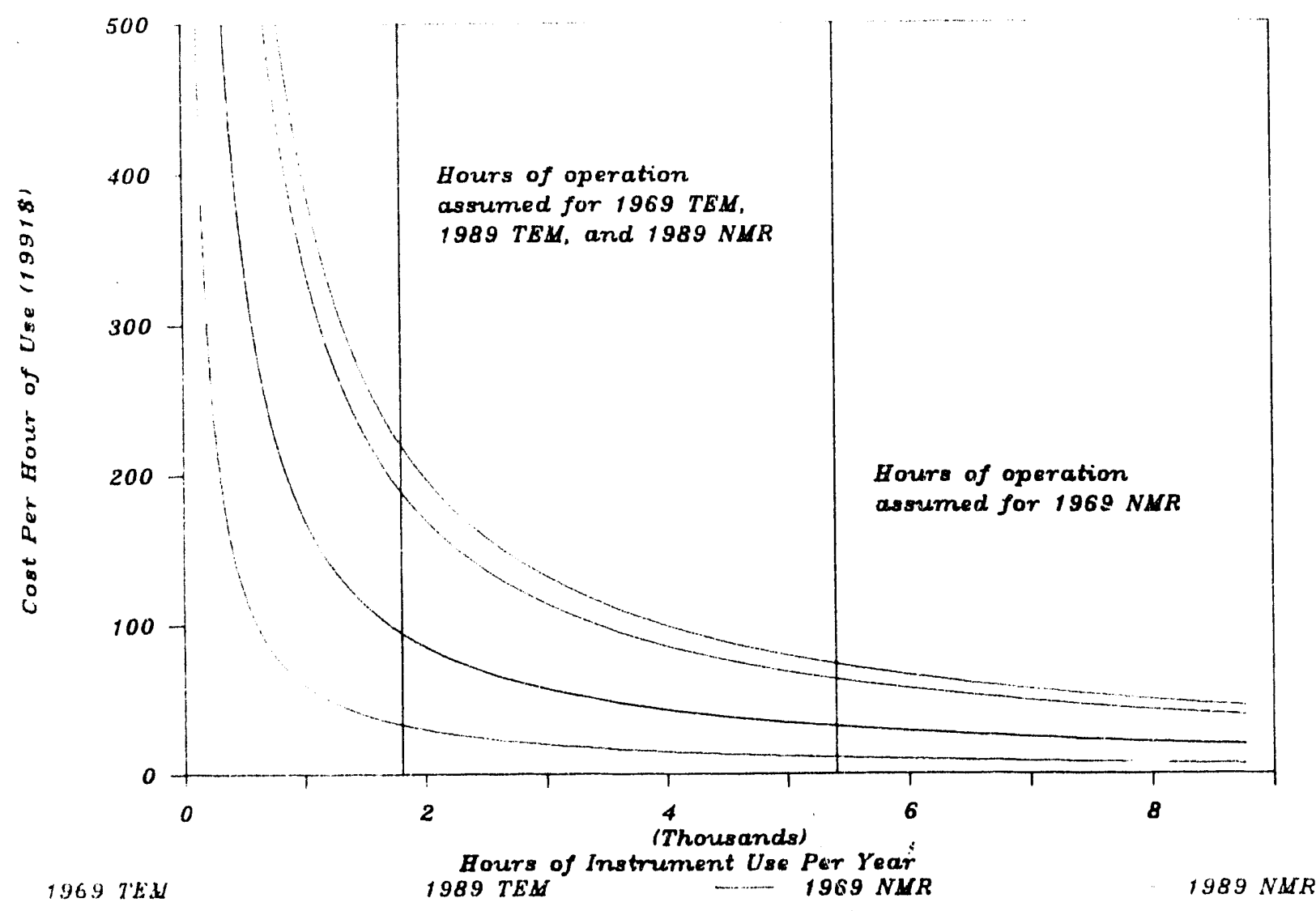

Figure 7. Cost of Instrument Use Versus Hours of Operation

instead of 2000). The decrease in time required to conduct an experiment means that many more experiments per year must be conducted with a state-of-the-art instrument in order to maximize its effectiveness.

In the future, the cost of sophisticated instruments will most likely continue to increase with the rapid pace of instrument advances, while the required instrument time for a given experiment will continue to decrease. The high cost and short lifetimes of these leading-edge, sophisticated instruments means they will have to be used more efficiently. A single user will find it difficult to adequately use or justify the cost of replacing state-of-the-art equipment.
Because the instruments can perform many more experiments annually than their historical counterparts, centralized user facilities containing a diversity of state-of-the-art instrumentation are recommended as a cost-effective strategy for reducing the cost of scientific investigations. User facilities make sense because ultrafast instruments on the horizon will provide scientists with so much data so fast that it could take months to interpret and apply the information. When coupled with computer networks such as NSFnet, user facilities will be accessible to everyone (even from off-site locations). The central laboratory will become a workstation that will allow scientists to create needed interfaces between multiple instruments, computers, and databases. 


\section{Conclusions}

Synergistic advancements in ab initio simulation, computation, and instrumentation are resulting in unprecedented opportunities for increasing the productivity of experimental science. However, two collaborative approaches are necessary for science to fully exploit emerging scientific opportunities.

First, the high cost and short life cycle of emerging instruments and computers make it necessary to allocate resources to an infrastructure that facilitates a coordinated, centralized research approach. The cost-effective laboratory of the future is a central "user facility" with highly automated instruments, state-of-theart computational tools, specialized staff to operate and maintain equipment, and network access for the individual investigator to both instruments and supercomputers.

Second, an exploratory research approach that couples experimental probes, massively parallel processing, and $a b$ initio simulation is needed. This integrated approach will enable the development of computational tools that provide a theoretical framework to guide the development of new materials and processes. The productivity of experimental science, as it strives to produce innovations that result in new and improved products and processes, maintain U.S. industrial competitiveness, and clean up the environment, will greatly increase as a result of the increased cost-effectiveness of research commensurate with these two strategies.

\section{References}

Baum, R. M. September 19, 1988. "Synchrotron Radiation Sources to Provide New Probes of Matter." Chemical and Engineering News, pp. 22-26.
Berlin, F. B. 1987. "Supercomputers: A Policy Opportunity." Supercomputers: A Key Scientific, Technological, and Industrial Preeminence, J. R. Kirkland and J. H. Poore, eds. Praeger Publisher, New York.

Blake, C.C.F., D. F. Koenig, G. A. Mair, A.C.T. North, D. C. Phillips, and V. R. Sarma. 1965. "Structure of Henegg-white Lysozyme: A Three-Dimensional Fourier Synthesis at $2 \AA$ Resolution." Nature 206:757.

Branscomb, L. M. 1986. "Science in 2006." American Scientist 74:650-658.

Broach, R. W., R. A. Eades, J. J. Low, T. L. Barr, and J. W. Frazer. October 1986. Assessment of Theoretical and Experimental Tools for Applied Research and Exploratory Development in Certain Energy Technologies. Allied-Signal Engineered Materials Research Center, Des Plaines, Illinois.

Buzbee, B. L., and D. H. Sharp. 1985. "Perspectives on Supercomputing." Science 227(4687):591-597.

Dertouzos, M. L. 1986. "Harnessing Computers Together." Technology Review 89(2):4457.

Dongarra, J. J. 1985. Performance of Various Computers Using Standard Linear Equations Software in a Fortran Environment. ANL/ MCS-TM-23, Argonne National Laboratory, Argonne, Illinois.

Eberhardt, J. J., P. J. Hay, and J. A. Carpenter, Jr. 1986. "Materials by Design: A Hierarchical Approach to the Design of New Materials." Materials Research Society Symposia Proceedings 63:191-206. 
Fassbender, L. L., R. K. Sen, L. Keay, and J. J. Eberhardt. 1989. "Catalysis-by-Design: A Coordinated Approach to Catalyst Research and Development." PNL-SA-17246, Pacific Northwest Laboratory, Richland, Washington.

Gille Associates. 1961. Data Processing Exuipment Encyclopedia Electronic Devices. Detroit, Michisan.

Greene, B. I., R. B. Hochstrasser, R. B. Weisman, and W. A. Eaton. 1978. "Picosecond Transient Absorption Spectra of Hemoglobin." International Symposium Frontiers of Biological Energetics 2:10671073.

Hansma, P. K., V. B. Elings, O. Marti, and C. E. Bracker. 1988. "Scanning Tunneling Microscopy and Atomic Force Microscopy: Application to Biology and Technology." Science 242:209-216.

Iijima, S. 1971. "High Resolution Electron Microscopy of Crystal Lattice of TitaniumNiobium Oxide." Journal of Applied Physics 42:5891-5893.

Kay, L. E., G. M. Clore, A. Bax, and A. M. Gronenborn. 1990. "Four Dimensional Heteronuclear Triple-Resonance NMR Spectroscopy of Interleukin - 1 Beta in Solution." Science 249(4967):411-414.

Lazou, C. 1986. Supercomputers and Their Use. Oxford University Press, New York.

Maize, K. January 11, 1988. "Synchrotrons, Supercomputers Battle the Common Cold." New Technology Week 5:20.
National Research Council. 1988. The Impact of Supercomputing Capabilities on U.S. Materials Science and Technology. Publication NMAB-451, National Academy Press, Washington, D.C.

Randall, J. E. January 1988. "NMR: The Best TTning Since X-Rays?" Technology Review, pp. 59-65.

Reich, H. J., M. Jautelat, M. T. Messe, F. J. Weigert, and J. D. Roberts. 1969. "Nuclear Magnetic Resonance Spectroscopy. Carbon-13 Spectra of Steroids." Journal American Chemical Society 91:7445-7454.

Rush, J. E. 1985. "Computer Hardware and Software in Chemical Information Processing." Journal of Chemical Information Computing Science 24(3):140-149.

Williams, D.A.R. 1986. Nuclear Magnetic Resonance Spectroscopy, ACOL Series. John Wiley \& Sons, New York.

Williams, M. R. 1985. A History of Computing Technology. Prentice-Hall, Inc., Englewood Cliffs, New Jersey.

Zewail, A. H., and R. B. Bernstein. November 7, 1988. "Real-Time Laser Femtochemistry." Cherical and Engineering News, pp. 24-43. 

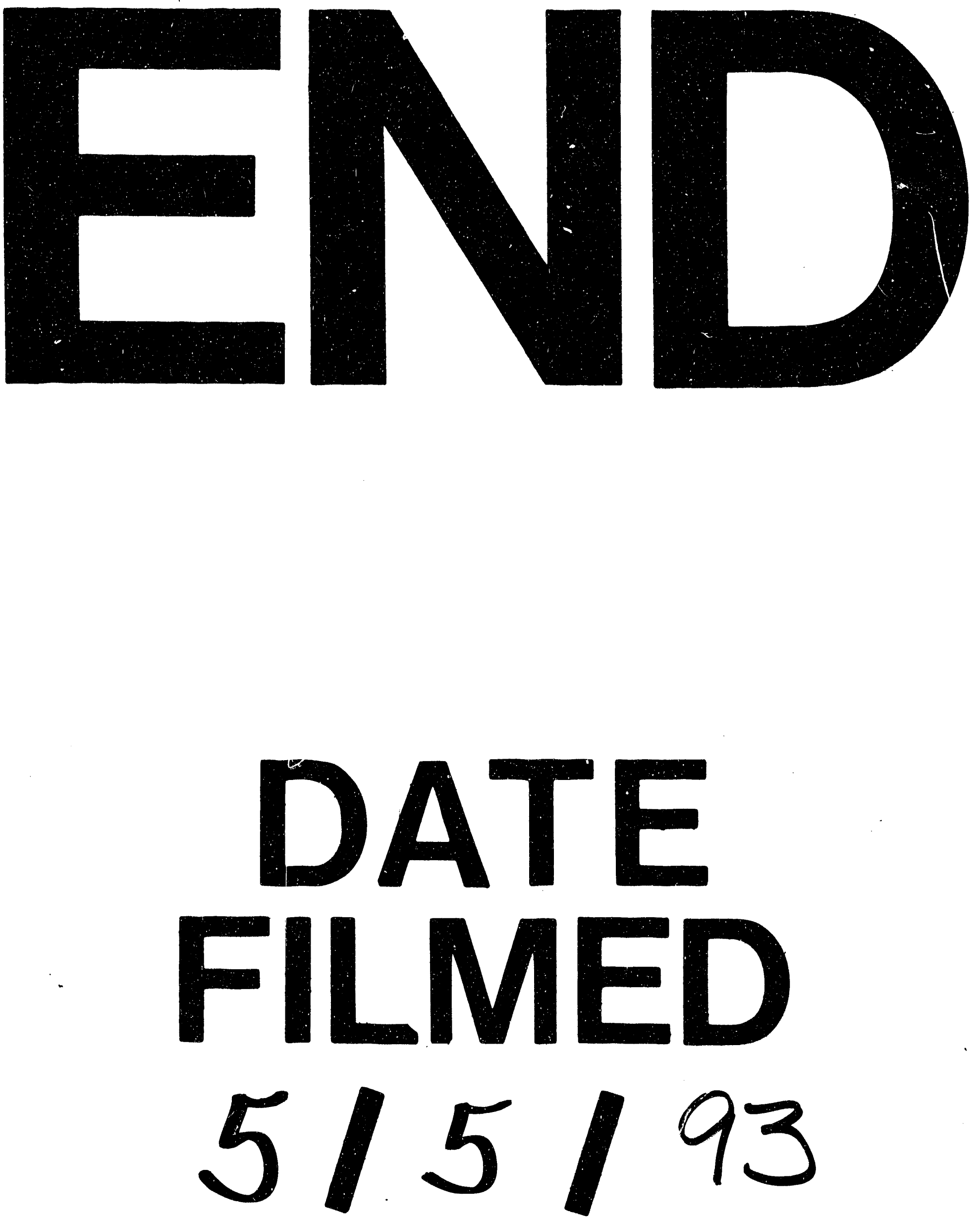
